\title{
H-GAP: Estimating Histograms of Local Variables with Accuracy Objectives for Distributed Real-Time Monitoring
}

\author{
Dan Jurca and Rolf Stadler
}

\begin{abstract}
We present H-GAP, a protocol for continuous monitoring, which provides a management station with the value distribution of local variables across the network. The protocol estimates the histogram of local state variables for a given accuracy and with minimal overhead. H-GAP is decentralized and asynchronous to achieve robustness and scalability, and it executes on an overlay interconnecting management processes in network devices. On this overlay, the protocol maintains a spanning tree and updates the histogram through incremental aggregation. The protocol is tunable in the sense that it allows controlling, at runtime, the trade-off between protocol overhead and an accuracy objective. This functionality is realized through dynamic configuration of local filters that control the flow of updates towards the management station. The paper includes an analysis of the problem of histogram aggregation over aggregation trees, a formulation of the global optimization problem, and a distributed solution containing heuristic, tree-based algorithms. Using $S U M$ as an example, we show how general aggregation functions over local variables can be efficiently computed with H-GAP. We evaluate our protocol through simulation using real traces. The results demonstrate the controllability of H-GAP in a selection of scenarios and its efficiency in large-scale networks.
\end{abstract}

Index Terms-Real-time monitoring, distributed aggregation, adaptive protocols.

\section{INTRODUCTION}

$\mathbf{T}$ HE ability to provide continuous estimates of management variables is vital for many management tasks, including network supervision, quality assurance, and proactive fault management. Often, management variables that are monitored in these tasks are aggregates that are computed from local device variables across the network. Example aggregates are the total number of VoIP flows, the maximum link utilization, the histogram of the current load across routers, or the distribution of the router out-degree in a network domain.

Engineering continuous monitoring solutions for network management involves addressing the fundamental trade-off between accurate estimation of a variable and the management overhead in terms of traffic and processing load [1],

Manuscript received DATE; revised DATE. The associate editor coordinating the review of this paper and approving it for publication was NAME.

The authors are with the Royal Institute of Technology (KTH), Laboratory for Communication Networks LCN, SE-100 44 Stockholm, Sweden (e-mail: \{danjurca, stadler\}@kth.se).

D. Jurca is currently with NTT DOCOMO Eurolabs in Munich, Germany.

This work has been part of 4WARD, a 7th Framework ISP project funded by the EC. It has further been supported by the ACCESS Linnaeus Center at KTH.

Digital Object Identifier 10.1109/T-WC.2008.I8P0292.
[2]. Network management solutions deployed today usually provide only qualitative control of the accuracy and do not support the setting of an accuracy objective. In the context of a monitoring system, setting accuracy objectives implies that the system exposes a "management knob" that controls the level of accuracy at which the system produces an estimate of the network state. The idea is that the monitoring system achieves such a level of accuracy with minimum overhead.

Distributed solutions for the aggregation of local variables usually push the aggregation function inside the network and aggregate the partial result in a decentralized way, e.g., [1], [3]. Such solutions prove to be efficient and exhibit good load balancing properties, along with increased robustness and resilience to network dynamics. However, they are individually tailored for the specific envisioned functionality and cannot be used in case the management station switches the objective function. E.g., a single instantiation of a distributed protocol designed for aggregating $S U M$ cannot provide an answer for a $M A X$ or $M I N$ query at the management station, hence a new instantiation of a different protocol is required. With this respect, the design of a distributed protocol that provides the management station with an accurate estimate of the histogram of the monitored local variable represents a worthy goal. Based on the estimate obtained through a single protocol instantiation, the management station has the flexibility of applying locally any desired aggregation function $\mathcal{F}$, either sequentially, or in parallel. This allows for instance $S U M$, $M I N$ and $M A X$ queries to be answered at the same time, only through local manipulation of the aggregate histogram.

In this paper, we address the problem of continuous monitoring of a local variable's histogram with accuracy objectives for large-scale network environments. Our goal is to design an efficient aggregation protocol that allows us to control the trade-off between the accuracy of the estimation and the protocol overhead. Our protocol continuously computes the distribution of the values of a monitored local variable through histogram aggregation by (i) creating and maintaining a selfstabilizing spanning tree and (ii) incrementally aggregating the variable's histogram along the tree (Figure 1). It is pushbased in the sense that changes in the partial distribution of the monitored variable values are pushed from the leaf nodes towards the management station along the aggregation tree. The protocol controls the management overhead by filtering updates that are sent from nodes to the management station, by allowing for local errors between the last sent updates and 


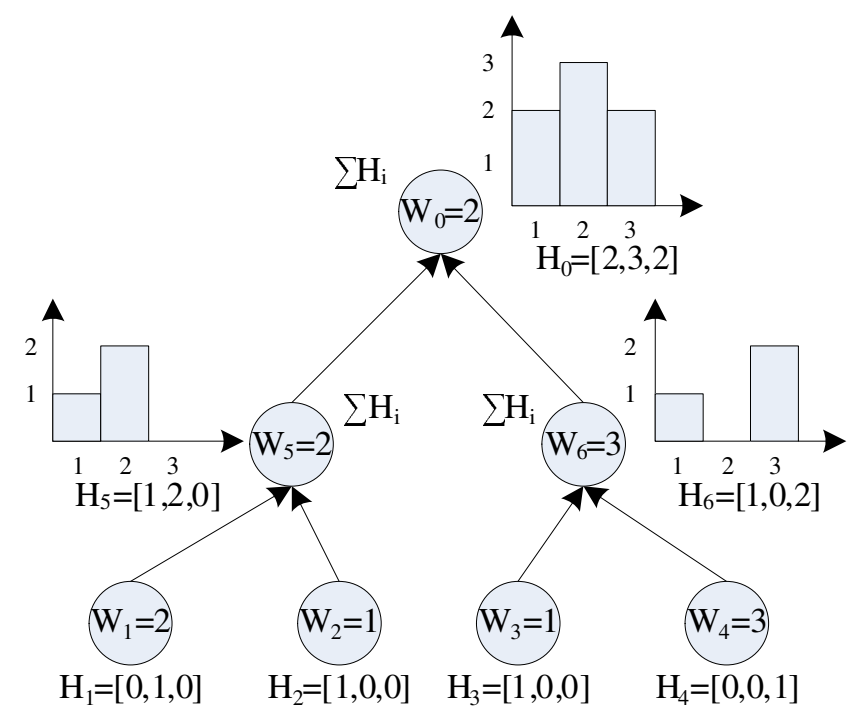

Fig. 1. Histogram Aggregation on Trees: local values $w_{i}(t) \in S$, where the value interval $S=\{1,2,3\}$. The aggregation vectors $H_{i}$ represent the partial aggregated histogram of the local values $w_{j}$ of nodes $j$ belonging to subtree $T_{i}$. Intermediate nodes in the tree aggregate the received vectors $H_{i}$ through element-wise sum.

the current network status.

The local error filters periodically adapt to the dynamics of the monitored variables and the network environment, observing a global error objective for the monitoring process. All operations in our protocol, including the incremental computation of the histogram and the configuration of the local error shares, are executed in a decentralized and asynchronous fashion to ensure robustness and achieve scalability.

While designing our protocol we keep in mind the goals of (i) controllable accuracy, in the sense of controlling the trade-off between estimation accuracy and protocol overhead, (ii) dynamic adaptation to changes in the network topology, or changes in the evolution of local variables, and controllability of this adaptation process, and (iii) scalability in terms of marginal protocol overhead increase with the system size.

The novelty and contributions of this paper are four-fold:

- We present a novel network protocol for distributed histogram aggregation with accuracy objectives using aggregation trees. The challenge is to dynamically compute the local filters, which we approach through solving a global optimization problem using distributed and efficient heuristics. To the best of our our knowledge, we are the first to present such a protocol;

- We formalize the trade-off between protocol overhead and accuracy objective, and we provide an analysis of this trade-off based on a simple stochastic model for the evolution of the local variables;

- We discuss how a general aggregation function $\mathcal{F}$ can be estimated for a given accuracy objective using H-GAP. We compare our solution based on H-GAP for the case of the $S U M$ aggregation function, with a state-of-the-art protocol that computes the sum inside the network.

- We assess through simulations to which extent our protocol meets the above design goals, with respect to controllability and scalability.
The paper is organized as follows: We address the related literature in Section II, while in Section III we introduce our system model and the notations used throughout the paper. We offer a short overview of our protocol in Section IV. The global optimization problem is presented in Section V, while Section VI attempts its full mathematical modelling in a centralized architecture. We present our distributed heuristic algorithms in Section VII and Section VIII, and analyze their performance in Section IX. Finally we discuss our contributions and future work in Section X, and we conclude our paper in Section XI.

\section{RELATED WORK}

Most research in aggregation protocols for monitoring is currently conducted in the context of wireless sensor networks, and we refer to some relevant work in this area below. In terms of more traditional networking environments, Astrolabe [4] is an example of a commercially deployed aggregation protocol for network monitoring. In contrast to H-GAP, the aggregation tree in Astrolabe has to be configured manually. Furthermore, Astrolabe does not support the use of accuracy objectives and thus, generally, incurs a higher overhead than H-GAP.

In the context of IP networks, existing works on monitoring aggregates focus on n-time queries, which request an estimation of the aggregate at discrete times. n-time queries are commonly realized as periodic and independent 1-time queries, which repeatedly perform a state snapshot [3], [5][9]. The trade-off between accuracy and management traffic is usually controlled through the sampling rate. [10] presents a solution for computing optimal sampling rates. While, at first sight, similar in functionality to the above works, our proposed protocol, H-GAP, implements a push-based approach to monitoring, which allows to continually estimate a network aggregate. H-GAP is therefore qualitatively different from a pull-based protocol that performs monitoring through a series of snapshots.

In large-scale networks, continuous monitoring of aggregates with high accuracy is often unfeasible due to high management traffic and processing overhead. Consequently, when designing monitoring protocols, the engineering tradeoffs must be understood. Much work in this direction has recently been performed in the context of wireless sensor networks. Several works investigate the trade-off between latency for computing an aggregate and either management traffic or energy consumption [11]-[14]. A different tradeoff is that of accuracy versus management traffic (e.g., [2], [3], [15]-[17]), where the focus is on providing guarantees on the accuracy of the estimation, ensuring that the difference between the estimation and the actual aggregate remains within a configurable range.

The trade-off between accuracy and overhead for continuous queries is also discussed in [18]. The authors propose a centralized design [18]-[20] where all nodes report directly to the management station, which aggregates the updates. In contrast, our protocol, H-GAP, applies incremental aggregation, which (i) distributes the cost of computing the aggregate among all nodes, and (ii) permits local value changes to cancel each other out, thus reducing the overhead. Different instantiations of this 
trade-off, namely, latency vs. overall traffic and accuracy vs. storage requirements, have been investigated in [21], [22].

Previous work on monitoring local network variables using aggregation trees is presented in [3] and [1]. The authors propose aggregation protocols with accuracy objectives for a single aggregation function, i.e., $S U M$. Both works compute the desired aggregate incrementally along a tree, and they use local filters to reduce the computational overhead. The first work uses synchronized rounds of operation, the second represents a fully distributed, asynchronous solution. While following the second approach, our work is more general, as through histogram aggregation we allow the management station to locally compute a range of functions on the aggregated data. Our protocol is fully decentralized as in [1], with the difference that the complexity of the filter computation is much lower.

Probably the closest work to our proposed protocol is presented in [2], which considers the aggregation of frequency distributions. Examples considered by the authors for such distributions include the number of flows per application and the number of flows per destination. The authors express the error objective in terms of the quantization of the frequency distribution and the update frequency. The considered aggregation scenario is a one-level hierarchy, where local stations communicate directly to the manager. In addition, the authors sketch the idea of aggregating frequency-distributions in hierarchical systems using aggregation trees, similar to our design. However, they do neither propose nor analyze a protocol solution for hierarchical scenarios, and they do not present any evaluation.

In [23]-[25] the authors utilize gossip algorithms to obtain an estimate of the value distribution at any node in the network. These works follow a very different approach to monitoring than to our solution does, for which, until now, the support of accuracy objectives has not been shown.

\section{System Model AND Notation}

\section{A. System Architecture}

We assume a distributed management architecture, where each network device participates in the monitoring task by running a management process. These management processes communicate via a management overlay network for the purpose of monitoring. We refer to this overlay as the timevarying network graph $G(t)=(V(t), E(t))$. A node $n \in V(t)$ represents a management process.

We assume that the network graph contains $N$ management processes, and is organized as an aggregation tree $T_{0}$, rooted at the monitoring process 0 . (We actually require from the network graph only that it is connected. On top of that graph, H-GAP will create and maintain a spanning tree, as described in Section IV. That tree is then used for incremental aggregation. ) Let $T_{n}$ be the subtree rooted a process $n \leq N$, and let each process $n$ run one leaf node and one aggregating node of this tree.

Each leaf $n$ has an associated local variable $w_{n}(t)$ which is an integer-valued quantity. The term local variable is used to represent a local state variable or device counter that is being subjected to monitoring. Local variables are updated asynchronously with a given sampling rate. Without loss of generality, assume that $w_{n}(t) \in S=\left\{s_{1}, \ldots, s_{m}\right\}, s_{i} \in \Re^{+}$, where $S$ is the finite set of all possible values that can be taken by $w_{n}(t), \forall n \leq N, \forall t$. Finally, let $\mathcal{P}$ be the stochastic process associated with the evolution of the local variables over time.

\section{B. Histogram Definitions}

Let $H_{n}(t)$ be the aggregation vector representing the histogram of all local values belonging to nodes in subtree $T_{n}$. If $H_{n}^{k}(t)$ denotes the number of local variables with value $w_{j}(t)=s_{k}, j \in T_{n}$, then $H_{n}^{k}(t)=\sum_{j \in T_{n}} c_{j, k}(t)$, where:

$$
c_{j, k}(t)= \begin{cases}1 & \text { if } w_{j}(t)=s_{k} \\ 0 & \text { otherwise }\end{cases}
$$

Then we can compute the aggregation vector $H_{n}(t)=$ $\left[H_{n}^{1}(t), \ldots, H_{n}^{m}(t)\right]$, with $m=|S|$. Observe that the aggregation vector of a leaf node has a value of 0 on all vector positions, except on the position corresponding to its local variable, where it has a value of 1 .

We define the distance between two aggregation vectors of the same length $m, H_{i}$ and $H_{j}$, as the total number of differences in the aggregated local values:

$$
D\left[H_{i}, H_{j}\right]=\sum_{k=1}^{m}\left|H_{i}^{k}-H_{j}^{k}\right| .
$$

Note that our defined distance $D\left[H_{i}, H_{j}\right]$ satisfies the formal necessary conditions for any distance metric: positive definiteness, symmetry and the triangle inequality [26].

At the same time we define the $y$-distance between the aggregation vectors as $D_{y}$ (e.g., $D\left[H_{i}, H_{j}\right]=y$ ), and $P_{y}=$ $\operatorname{Prob}\left(D\left[H_{i}, H_{j}\right]=y\right)$ as the corresponding probability of having a distance $D_{y}$ between two aggregation vectors. Note that as long as the number of aggregated local values stays constant in time, $P_{y}=0$ for all odd numbers $y$, which means that a single variable having different values in the two histograms leads to a distance $D_{2}$ between the histograms. Similarly, if $d$ is the number of variables aggregated in histograms $H_{i}$ and $H_{j}$, the maximum distance between the histograms (which is the case when none of the $d$ values overlap) is $D_{2 d}$, which is equivalent

to $P_{y}=0, \forall y>2 d$. $P_{y}$ depends on the stochastic process $\mathcal{P}$ characterizing the change of local variable values over time.

Let $\epsilon(t)$ be the error at the management station (or root process 0 ), expressed as the distance between the aggregated vector $H_{0}(t)$ available at the root as a result of the aggregation protocol, and the actual histogram of all local values in the network at time $t^{1}$. Finally we define $\epsilon_{M}$ as the error objective of our monitoring system, which translated into an inverselyproportional accuracy objective. $\epsilon_{M}$ influences the overhead of the aggregation protocol, as a large tolerated error allows for less frequent vector updates, hence reduced protocol overhead, while a smaller tolerated error imposes more frequent protocol updates, as local values change over time.

\footnotetext{
${ }^{1}$ By actual histogram we mean the histogram of the local values that can be theoretically computed if all variables are known at a given time.
} 


\section{Aggregation Protocol Notations}

The aggregation protocol sends message updates from children processes to their parents in order to aggregate the histogram of the local values at each level in the tree rooted at the management station (Figure 1). An update message sent by node $n$ contains the aggregation vector $H_{n}(t)$ available at node $n$ at time $t$. A parent process $n$ having the set of child processes $\gamma(n)$ (including its own leaf node) aggregates the received update messages and computes the latest histogram of its subtree:

$$
H_{n}(t)=\sum_{j \in \gamma(n)} H_{j}(t) .
$$

Here, by $H_{j}(t)$ we denote the most recent aggregation vector available at parent node $n$ through the last message update received from child node $j$. The sum is computed as the element-wise sum of the elements $H_{j}^{k}$.

The update rate of child process $j \in \gamma(n)$ is denoted by $R_{j}^{\text {out }}(t)$ and is computed as the average number of update messages sent towards parent $n$ in a time window. $R_{j}^{\text {out }}(t)$ depends on the relative change in monitored values (compared to the last update sent) and on the error share $\epsilon_{j}(t) . \epsilon_{j}(t)$ is allocated by the protocol for each individual process $j$, as a share of the total tolerated error $\epsilon_{M}$. Furthermore, the rate of incoming updates at parent node $n$ is computed as the sum of incoming update rates: $R_{n}^{i n}(t)=\sum_{j \in \gamma(n)} R_{j}^{o u t}(t)$. Finally, the overhead at node $n$ is computed as $\Omega_{n}(t)=R_{n}^{i n}(t)+$ $R_{n}^{\text {out }}(t)$.

We observe that $R_{j}^{\text {out }}(t)$ and $\Omega_{n}(t)$ are functions of (i) the stochastic process $\mathcal{P}$ modeling the changes in local values at all processes $j$ belonging to subtree $T_{n}$ (including process $n$ ), and (ii) their associated error shares $\epsilon_{j}(t)$. Therefore, $R_{j}^{\text {out }}(t)=R_{j}^{\text {out }}\left(t, \mathcal{P}, \epsilon_{j}(t)\right)$ and $\Omega_{n}(t)=\Omega_{n}\left(t, \mathcal{P}, \epsilon_{j}(t)\right)$, $\forall j \in T_{n}$. While $R_{j}^{\text {out }}$ and $\Omega_{n}$ characterize only the number of messages exchanged by our protocol (and not the total bitrate required), they are an appropriate measure for the total protocol overhead, as long as the message size is kept constant. This is in fact the case, as long as the size of the update vector is constant at all processes $n$, i.e., the cardinality of the value set $|S|$ remains constant at all processes. All definitions and notations used throughout this paper are summarized in Table I.

\section{Protocol Overview}

Our objective is to engineer a protocol on the network graph that provides a management station with a continuous estimate of the histogram of the local values, for a given accuracy objective. The protocol should execute with minimal overhead, expressed as the number of updates processed per second in the network. The accuracy is expressed as the maximum error of the estimate over time.

The design objectives of our protocol are three-fold:

- controllable overhead-accuracy trade-off: by considering the accuracy objective as a control parameter, the overhead of our protocol should vary as a function of the required performance in terms of aggregate accuracy.
TABLE I

DEFINITIONS AND NOTATIONS

\begin{tabular}{|c|c|}
\hline Total number of nodes in the network & $N$ \\
\hline Local weight at node $n$ & $w_{n}$ \\
\hline Value set for local weights & $S=\left\{s_{1}, \ldots, s_{m}\right\}$ \\
\hline Generic stochastic process for local weight change & $\mathcal{P}$ \\
\hline Local histogram at node $n$ at time $t$ & $H_{n}(t)$ \\
\hline Histogram distance metric & $D\left[H_{i}, H_{j}\right]$ \\
\hline$y$-distance between two histograms & $D_{y}$ \\
\hline Probability of $D_{y}$ & $P P_{y}$ \\
\hline Sub-tree rooted at node $n$ & $T_{n}$ \\
\hline Set of children of node $n$ & $\gamma_{n}$ \\
\hline Maximum error objective & $\epsilon_{M}$ \\
\hline Error-share at node $n$ at time $t$ & $\epsilon_{n}(t)$ \\
\hline Error-share allocation vector at time $t$ & $\epsilon(t)$ \\
\hline Outgoing update rate at node $j$ at time $t$ & $\overline{R_{j}^{\text {out }}(t)}$ \\
\hline Incoming update rate at node $n$ at time $t$ & $R_{n}^{i n}(t)$ \\
\hline Protocol overhead at node $n$ at time $t$ & $\Omega_{n}(t)$ \\
\hline Error-share minimum step & $\Delta \epsilon$ \\
\hline Update rate threshold & $\delta R$ \\
\hline Management function applied on $H$ & $\mathcal{F}(H)$ \\
\hline Result of $\mathcal{F}(H)$ & $\mathcal{R}_{\mathcal{F}}$ \\
\hline Error of $\mathcal{R}_{\mathcal{F}}$ & $\epsilon \mathcal{F}$ \\
\hline Set of outdated values in $H$ & $O_{k}$ \\
\hline Proportionality factor between $\epsilon_{M}$ and $\epsilon_{\mathcal{F}}$ & $f_{\max }$ \\
\hline Maximum weight-change per update interval & $\Delta w$ \\
\hline
\end{tabular}

- dynamic adaptation to changes: the algorithm should adapt in real-time both to changes in the network topology and to the evolution of the local variables. Furthermore, the adaptation process should be controllable;

- scalability: we design our protocol for large, dynamic systems, in such a way that its overhead increases marginally with the system size;

We call our protocol for histogram aggregation H-GAP. The protocol is based on GAP (Generic Aggregation Protocol), developed in our earlier work [27]. GAP is an asynchronous distributed protocol that builds and maintains a BFS (Breadth First Search) spanning tree on an overlay network, in a similar way as the algorithm that underlies the 802.1d Spanning Tree Protocol [28].

In GAP each node holds information about its children in the BSF tree, in order to compute the partial aggregate, i.e., the aggregate value of the local management variables from all nodes of the subtree where this node is the root. GAP is event-driven in the sense that messages are exchanged as results of events, such as the detection of a new neighbor on the overlay, the failure of a neighbor, an aggregate update or a change in the local management variable.

A general problem experienced in tree-based aggregation schemes is that they can cause a high load on the root node or on nodes close to the root, specifically in large networks. In order to reduce this overhead, one can either apply a rate limitation scheme, which imposes an upper bound on message rates on each link, or one can introduce a filter scheme, whereby a node drops updates when only small variations of its partial aggregate occur [1]. In both cases, the protocol overhead is reduced at the cost of introducing an error in the aggregate estimation process.

For H-GAP, we choose an error filter scheme that forwards a message update from a node $n$ to its parent only when its current partial aggregate differs more than a given error threshold $\epsilon_{n}$ from the last reported aggregate. The filter 
scheme is the key extension we make to the GAP protocol. The functionality of GAP that is inherited by H-GAP is (i) creating and maintaining the aggregation tree (and specifically handling node arrivals, departures and failures) and (ii) performing the aggregation function on the tree using generic functions and data structures related to maintaining partial aggregates at parent nodes at different levels in the tree.

In the following sections we express our protocol objectives in the form of a global optimization problem and offer its full formalization. Afterwards, we provide heuristic algorithms which give greedy solutions in distributed and asynchronous network settings.

\section{Optimization PRoBlem}

Under the given system setup and notation, we want to derive an update protocol which computes in real time an estimate of the histogram of the monitored local values inside the network. We are interested in minimizing the maximum link overhead of such

protocol, while still fulfilling the accuracy objective imposed on our setup. Formally, we denote as $\epsilon \overrightarrow{(t)}=$ $\left[\epsilon_{0}(t), \ldots, \epsilon_{N}(t)\right]$ the vector of all error shares distributed by our algorithm to all processes in the management system at time $t$. We want to compute:

$$
\overrightarrow{(t)}^{*}=\underset{\forall(\epsilon(t))}{\arg \min }\left(\max _{\forall j \in T_{0}}\left(R_{j}^{\text {out }}\left(\epsilon_{j}(t)\right)\right)\right)
$$

such that the total error constraint is satisfied at all times:

$$
\epsilon(t) \leq \epsilon_{M}, \forall t
$$

Hence, the optimization problem translates into finding the optimal split of the total system tolerated error $\epsilon_{M}$ into local shares $\epsilon_{n}$. The underlying criterion behind the split insures the minimization of the total protocol traffic over the busiest network link. In the case of a real system, where its correct functioning requires all nodes to be alive, this criterion can be translated as maximizing the total network lifetime. At the same time, it can be perceived as a system fairness measure.

Notice that for a given fixed stochastic process $\mathcal{P}$, the total protocol overhead depends on all individual update rates at each process $n$ in the tree, which in turn depends on the allocated local shares of tolerated errors $\epsilon_{n}$.

We attempt a full formalization of the optimization problem under some strict assumptions and for a simple stochastic process for the local value evolution in time in the next section.

\section{ANALYSIS}

In this section we analyze the optimization problem for a simple update process $\mathcal{P}$ for all local variables $w_{n}(t)$. For the sake of simplicity, we assume that the network is synchronized, and that all local values change periodically with the same period $\tau$. We assume that at each time step, each process updates its local variable to a new value from $S$ according to a uniform distribution. Hence, this memoryless process yields at each time step, or current time $t_{c}$, local values $w_{n}\left(t_{c}\right)=s_{k}, \forall n \leq N, \forall k \leq m$, where $m=|S|$, with probability:

$$
P\left(w_{n}\left(t_{c}\right)=s_{k}\right)=\frac{1}{m}, \forall t_{c}, \forall n, \forall k .
$$

Notice that updates of value $w_{n}$ do not depend on the specific time step in the runtime of the system. Hence, in our analysis we simplify our notations whenever possible, and do not refer to time anymore.

\section{A. Leaf Processes}

Leaf processes $n$ do not receive any incoming messages, as there is no subtree $T_{n}$ rooted at $n$. Hence $R_{n}^{i n}(t)=0, \forall t$. We assume that each leaf process $n$ sends a message update to its parent as soon as the local value $w_{n}$ changes value compared to the last sent update, e.g., $w_{n}\left(t_{c}\right) \neq w_{n}\left(t_{u}\right)$, or $\epsilon_{n}=0$. We denote the time of the last sent update as $t_{u}$. According to $\mathcal{P}$, this happens with probability $P\left(w_{n}\left(t_{c}\right) \neq w_{n}\left(t_{u}\right)\right)=1-\frac{1}{m}$. Hence the outgoing rate of message updates of a leaf process normalized with the time period is:

$$
R_{n}^{\text {out }}=\frac{m-1}{m},
$$

and the associated protocol overhead with leaf process $n$ is $\Omega_{n}=R_{n}^{\text {out }}$.

\section{B. Aggregating Processes}

An aggregating process $n$ is the root node of the subtree $T_{n}$, and receives update messages from all its children $j \in \gamma_{n}$. The incoming rate perceived by process $n$ is $R_{n}^{\text {in }}=\sum_{j \in \gamma_{n}} R_{j}^{\text {out }}$, where $R_{j}^{\text {out }}$ can be the outgoing message rate of a leaf process or another aggregating process.

$R_{j}^{\text {out }}$ of an aggregating process $j$ can be computed as the probability of a distance larger than $\epsilon_{j}$ between the last sent update and the current aggregation vector. For notation simplification we denote as $D(j)=D\left[H_{j}\left(t_{c}\right), H_{j}\left(t_{u}\right)\right]$. Under our model $\mathcal{P}$, this probability is invariant in time, hence we have:

$$
R_{j}^{\text {out }}=\operatorname{Prob}\left(D(j)>\epsilon_{j}\right) .
$$

Let $P^{1}=\operatorname{Prob}\left(D(n)>\epsilon_{n}\right)$. Then we can compute:

$$
P^{1}=\sum_{y=\epsilon_{n}+1}^{2 d(n)} P_{y}(n),
$$

where $P_{y}(n)$ is the probability of a $y$-distance between two aggregating vectors $H_{n}\left(t_{c}\right)$ and $H_{n}\left(t_{u}\right)$ as defined in the previous sections, and $d(n)$ is the size of the subtree $T_{n}$ rooted at process $n$.

$P_{y}(n)$ is computed at process $n$ as the distance between the last update vector sent up on the tree and the newly computed aggregated vector, as summed from message updates received from children. It depends on the individual distances reported in each of the update vectors received from children, taking into account that in the final aggregated vector $H_{n}$ distances from different children vectors $H_{j}$ might cancel each other out. 
If $c(n)=\left|\gamma_{n}\right|$ is the number of direct children of process $n$ in tree $T_{n}$, then $P_{y}$ can be recursively computed at process $n$ as:

$$
P_{y}(n)=\sum_{a_{1}=1}^{2 d(1)} \ldots \sum_{a_{c(n)}=1}^{2 d(c(n))}\left[P_{y}^{2}(n) \prod_{i=1}^{c(n)} P_{a_{i}}(i)\right],
$$

where $P_{y}^{2}(n)$ is the probability that the distance between the last updated vector $H_{n}\left(t_{u}\right)$ and the newly computed vector $H_{n}\left(t_{c}\right)$ is equal to $y$ (e.g., $D(n)=y$ ) given that the distance between the vectors from any child $j$ is $a_{j}$ (e.g., $D(j)=a_{j}$ ):

$$
P_{y}^{2}(n)=\operatorname{Prob}\left(D(n)=y \mid D(j)=a_{j}, \forall j \in \gamma_{n}\right) .
$$

$P_{y}^{2}$ can be numerically computed under the assumption of $\mathcal{P}$ knowing the size of the subtree $d(j)$ rooted at any child process $j$ of process $n$, starting from the binomial distribution of the probability of a distance $D_{k}$ out of a maximum possible distance $D_{2 d}: P_{2 d}(k)=\left(\begin{array}{c}2 d \\ k\end{array}\right) p^{k}\left(1-p^{2 d-k}\right)$, with $p=\frac{m-1}{m}$.

After computing $R_{n}^{\text {out }}$, a centralized algorithm that knows the set of children $\gamma_{n}$ of any process $n$ in the aggregation tree $T_{0}$ can compute the optimal vector allocation $\epsilon(\vec{t})^{*}$, and hence solve the optimization problem.

In the next section we present our heuristic algorithm that computes an approximation of the optimal error share allocation for all processes in the tree in a distributed way, with no assumption on full topology knowledge or stochastic process $\mathcal{P}$.

\section{Distributed Heuristic Solution}

This section details our heuristic solution for the distributed computation of local error shares $\epsilon_{n}$ which influence the overhead of H-GAP under the total error objective $\epsilon_{M}$. First we approximate the global optimization problem with local optimization problems that can be solved in a distributed way at all parent nodes in the tree. Then we provide a fast heuristic algorithm which offers a solution for the local problem.

\section{A. Distributed Optimization Problem}

Let process $n$ have an allocated error budget $\epsilon_{n}$ that must be distributed to all child processes in aggregation tree $T_{n}$. As before, let $c(n)=\left|\gamma_{n}\right|$ be the total number of direct children of process $n$ and let $d(n)$ be the size of $T_{n}$. We also denote as $\epsilon(\vec{n})=\left(\left\{\epsilon_{j} \mid \forall j \in \gamma_{n}\right\}\right)$ the allocation of error shares to all child processes. We want to compute:

$$
\epsilon(\vec{n})^{*}=\underset{\forall \vec{\forall}(\epsilon(n))}{\arg \min }\left(\max _{\forall j \in \gamma_{n}}\left(R_{j}^{\text {out }}\left(\epsilon_{j}\right)\right)\right),
$$

under the constraint: $\sum_{j \in \gamma_{n}} \epsilon_{j} \leq \epsilon_{n}$.

With a full knowledge of the behavior of partial aggregates from children, a full-search algorithm can yield the optimal vector $\epsilon(\vec{n})^{*}$, and hence solve the optimization problem. However, monitoring and estimating even on short time windows the evolution of the partial children aggregates requires a high volume of information exchange and storage [3], which in the end might defeat the purpose of our solution. Hence, in the rest of this paper we concentrate on a solution based on a faster greedy algorithm which scales even for large network systems.

\section{B. Protocol Initialization}

H-GAP initializes by constructing a spanning tree on the management overlay in the same way as GAP does [27]. On all nodes of the aggregating tree the error shares are initialized to zero, so that all changes in the partially aggregated histograms are reported up the aggregation tree to the root node. During a warm-up period, each parent node $n$ monitors the incoming message rate from all its children and finds the child node that loads the network most, by sending the largest number of updates. After the warmup period the accuracy objective $\epsilon_{M}$ is set at the root node and the protocol starts running in a distributed way.

\section{Overhead Estimation}

Each parent node estimates the overhead for each of its children, in order to identify at all times the child that induces the largest protocol overhead. We achieve this by maintaining at the parent node, for each of its children, a circular buffer which records the rate of child updates during a given monitoring window.

Let $R_{j}^{\text {out }}(t)$ be the rate of updates send by child node $j$ (e.g., the number of message updates sent by node $j$ towards its parent in a time unit), and let $T$ be the size of the monitoring window, measured in time units. Then, at any time $\tau$ the average rate estimate for child $j$ is: $\hat{R_{j}^{\text {out }}}=\frac{1}{T} \sum_{t=\tau-T}^{\tau} R_{j}^{\text {out }}(t)$, and the estimate of the total incoming rate as seen by parent $n$ is $\hat{R_{n}^{i n}}=\sum_{j \in \gamma_{n}} R_{j}^{\hat{o u t}}$.

The choice of the window size influences the performance of our algorithm. On the one hand, the variance of our estimator is inversely proportional to the window size. On the other hand, a short window size makes the system more adaptive.

\section{Heuristic $\epsilon \vec{n})$ Allocation Algorithm}

We present a polynomial-time algorithm as an efficient heuristic to find a solution vector $\epsilon(\vec{n})$ to Eq. (4). The computation is based on the step-wise optimization of our allocation of $\epsilon_{j}$. Our algorithm uses the child overhead estimation as presented above, in order to compute step-wise the child that induces the largest protocol overhead.

Starting from an initial error share allocation $\epsilon(\vec{n})$, at each iteration, the algorithm increases by $\Delta \epsilon$ the error share of the child that has the highest $R_{j}^{\hat{o u t}}$, in an attempt to reduce the protocol link overhead as seen by parent $n$ from child $j$. The iterations are performed with periodicity $t_{i}$, during which the parent monitors the effect of allocating the extra share $\Delta \epsilon$ to node $j$ and computes a crude estimate of its new $R_{j}^{\hat{o u t}}$. Notice that $R_{j}^{\hat{o u t}}$ can be monitored and roughly estimated on a time interval $t_{i}$ which is proportional to the sampling interval of the monitored local weights.

The algorithm terminates when the error budget $\epsilon_{n}$ is depleted, or when all child nodes become passive, i.e., their error share is large enough to prevent all aggregation updates towards the parent. The pseudo-code of the algorithm is presented in Algorithm 1. 


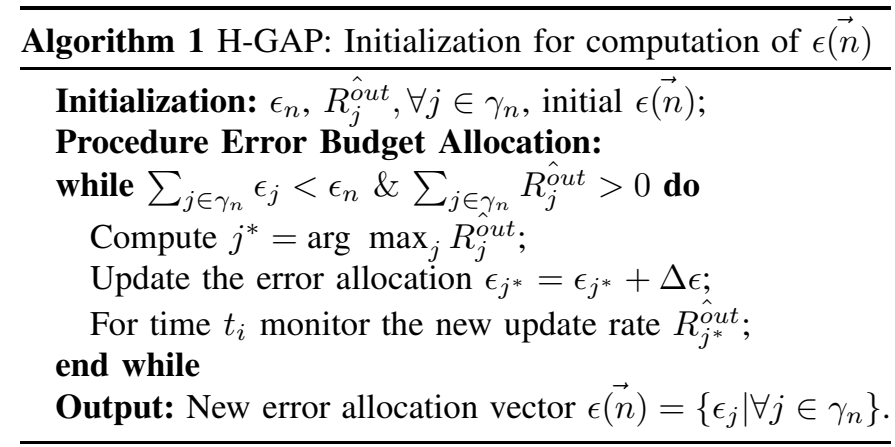

Algorithm 1 iteratively distributes the error budget $\epsilon_{n}$ to child processes, in a manner that attempts the minimization of their outgoing update rates. At each step, the algorithm increases the error budget of the child process that induces the highest protocol overhead. Recall that at system setup, when the algorithm is run for the first time, the initial error allocation vector is zero, e.g., $\epsilon(\vec{n})=0$.

At each iteration in the while loop, the algorithm searches the child $j^{*}$ with the maximum outgoing rate. Without any other computational or memory requirements during runtime, this operation requires $\left|\gamma_{n}\right|$ separate computations. The number of iterations is limited by the total error budget $\epsilon_{n}$. If $\epsilon_{n}$ is expressed as an integer number corresponding to the maximum tolerated distance between two aggregating vectors, and the minimum error share $\Delta \epsilon$ represents the constant minimum-increase step used by the algorithm, then the number of algorithm iterations is limited by $\frac{\epsilon_{n}}{\Delta \epsilon}$. Hence, the computational complexity of Algorithm 1 is $O\left(\epsilon_{n}\left|\gamma_{n}\right|\right)$.

The time needed by Algorithm 1 in order to converge to a stable solution at system initialization is limited by the maximum number of iterations, and can be computed as $\frac{\epsilon_{n}}{\Delta \epsilon} t_{i}$. A change of the error share of child $j$ initiated by parent node $n$ can be propagated in the whole subtree $T_{j}$ during the same protocol iteration, as valid rate estimates exist at all aggregating nodes in the subtree. Algorithm adaptation during system run-time is performed much faster, as presented next.

Observe that performing the optimization algorithms at all parent processes, starting at the root monitoring process with total error budget $\epsilon_{M}$ yields the local error shares at all processes, hence it offers a distributed, likely sub-optimal solution for the optimization problem presented in Section V.

\section{E. Dynamic $\epsilon(\vec{n})$ Re-computation}

Algorithm 1 runs at system initialization and computes an appropriate error share that is propagated along the aggregation tree, starting from the root down. As the behavior of local values changes in time, we need to adapt the error share allocation in order to reflect the new system status. This implies the redistribution of the error budget among children at every parent process.

The dynamic re-computation of the error allocation, locally at each process $n$, is event-driven and requires rerunning Algorithm 1. Events are represented by (i) a local reallocation decision taken by the parent in case its current incoming rate estimate $\hat{R_{n}^{i n}}$ is larger by $\delta R$ than the original incoming rate at the time of the last error share allocation, (ii) an increase of the parent error share $\epsilon_{n}$ by $\Delta \epsilon$, (iii) a decrease of $\epsilon_{n}$ by $\Delta \epsilon . \delta R$ is a control parameter of our protocol. A larger value implies less frequent reallocations, and hence a more stable system at the expense of a larger protocol overhead.

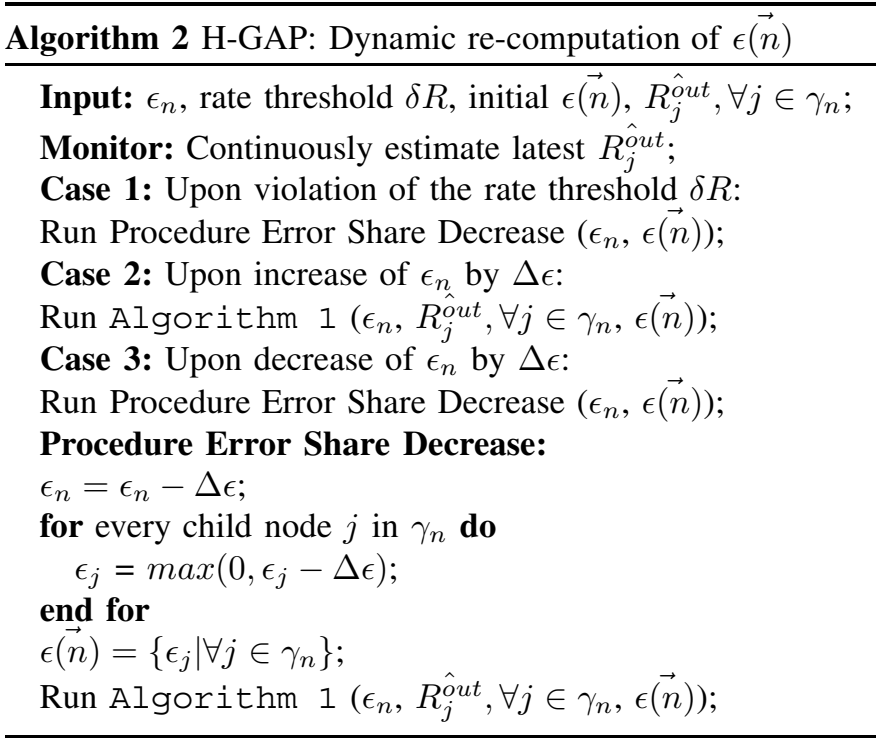

In case of event (ii), parent $n$ has enough information to reallocate the extra error share to its children by running an additional iteration of Algorithm 1 . However, for events (i) and (iii), observe that $\sum_{j \in \gamma_{n}} \epsilon_{j} \geq \epsilon_{n}$ and a reallocation of the shares automatically implies the reduction of the share of at least one child.

In these cases we apply the concept of error share shrinking [3] which implies that we reduce the error share of all children by $\Delta \epsilon$ such that under the new error allocation $\sum_{j \in \gamma_{n}} \epsilon_{j} \leq \epsilon_{n}$. For the established time window $t_{i}$ we recompute the estimates $R_{j}^{\text {out }}$ and we re-run Algorithm 1 starting with the new error allocation vector and the newly computed rate estimates for all children. We differentiate our method from [3] as we only apply the shrinking process locally, and event-based, e.g., only when needed. Hence, we expect our solution to be more efficient and more adaptive. A formalization of the above ideas is presented in Algorithm 2. Note that Algorithm 2 requires a single iteration run of Algorithm 1 for Case 2, while for Cases 1 and 3 the number of iterations is upperbounded by the number of children of process $n,\left|\gamma_{n}\right|$. Hence, the real-time adaptation of our mechanism is much faster than the initialization process, due to the smaller error share that must be reallocated.

\section{COMPUTING GENERAL AGGREGATION FUNCTIONS WITH ACCURACY OBJECTIVES USING H-GAP}

\section{A. Applying an aggregation function on the histogram}

In the previous sections, we presented the H-GAP protocol, which estimates the evolution of a histogram of local state variables. The protocol aims at keeping the estimation error, i.e., the distance between the protocol-generated histogram and the actual histogram, within the maximum error objective $\epsilon_{M}$. An intuitive interpretation of the error objective $\epsilon_{M}$ is the fraction of outdated, and therefore possibly incorrect, values represented in the histogram provided by H-GAP. For instance, 
an error objective of $x \%$ means that at most $x \%$ of the local values represented in the protocol-generated histogram are outdated.

Having an estimate of the histogram available at the monitoring node allows to locally compute estimates for a range of aggregation functions on that node. Such an aggregation function is of the form $\mathcal{F}\left(w_{1}, \ldots, w_{N}\right)$ with $\mathcal{F}(t)=$ $\mathcal{F}\left(w_{1}(t), \ldots, w_{N}(t)\right)$, and is symmetric in its arguments in the sense that any permutation of the arguments $w_{i}$ results in the same value of the function. The evolution of an estimate for this function $\mathcal{F}$ can be computed using the H-GAP generated histogram. Since H-GAP has an estimation error, the computation of $\mathcal{F}$ using the protocol generated histogram is associated with an error $\epsilon_{\mathcal{F}}$.

We are interested in estimating an aggregation function $\mathcal{F}$ with an error objective $\epsilon_{\mathcal{F}}$. Therefore, the problem becomes one of mapping $\epsilon_{\mathcal{F}}$ to the error objective of the H-GAP protocol $\epsilon_{M}$, which means finding a function $g$, such that

$$
\epsilon_{M}=g\left(\epsilon_{\mathcal{F}}\right)
$$

This mapping generally depends on the aggregation function $\mathcal{F}$. In the following subsection we present an example of such a mapping $g$ for the specific aggregation function $S U M$.

\section{B. Mapping the accuracy objective for $S U M$}

Using the histogram $H_{0}$ produced by H-GAP, the aggregation function $S U M$ can be computed at the monitoring node as:

$$
S U M\left(H_{0}\right)=\sum_{i=1}^{m} s_{i} H_{0}^{i},
$$

where $s_{i} \in S$.

Based on the discussion in Section VIII-A, the number of outdated values $k$ in the histogram $H_{0}$ can be computed as $k=\epsilon_{M} \cdot N$, where $\epsilon_{M}$ is normalized by the system size $N$.

Using $H_{0}, S, k$ and $S U M\left(H_{0}\right)$ one can compute the interval in which the actual value of $S U M$ must lie. As this interval can be very large in practice (e.g., for specific values $s_{i} \in S$ ) we need to restrict it for finding a relationship $g$ between $\epsilon_{M}$ and $\epsilon_{S U M}$ that allows for an efficient and effective estimation.

Let $\Delta w$ be the absolute change of the local values $w_{i}$ during a sampling interval $\Delta t$, averaged over all $w_{i}$ in the system. We express the error in estimating the actual value of $S U M$ by computing $S U M\left(H_{0}\right)$ as $k \cdot \Delta w$, which is the maximum of the average error between the two values over some monitoring time interval. (We implicitly assume here that the sampling interval $\Delta t$ is significantly larger than the computating and communication delays of the H-GAP protocol in the system. This assumption is reasonable for many technology domains, e.g., IP network environments.) This approach allows us to directly relate the changes in the local variables to the estimate $S U M\left(H_{0}\right)$ computed at the root node.

The experienced error in estimating the actual $S U M$ aggregation function, modelled as $k \cdot \Delta w$, can now be related to the error objective $\epsilon_{S U M}$ as follows:
TABLE II

Simulation PARAMETERS

\begin{tabular}{c|c}
\hline Value set $S$ & {$[1, \ldots, 124]$} \\
\hline Estimation Window & $30 \mathrm{~s}$ \\
\hline Algorithm Iteration Window $t_{i}$ & $1 \mathrm{~s}$ \\
\hline Error Step $\Delta \epsilon$ & 2 \\
\hline Variable sampling rate & $1 \mathrm{~s}$ \\
\hline Rate threshold $\Delta R=\frac{\delta R}{R_{n}^{i n}}$ & $20 \%$ \\
\hline Normalized Error Objective $\bar{\epsilon}$ & $\frac{D\left[H_{0}(t), H_{\text {real }}(t)\right]}{N}$ \\
\hline Protocol Initialization & $70 \mathrm{~s}$ \\
\hline Monitoring time & $100 \mathrm{~s}$ \\
\hline Error Computation Period & $0.5 \mathrm{~s}$ \\
\hline
\end{tabular}

$$
k \cdot \Delta w=\epsilon_{S U M} \cdot S U M\left(H_{0}\right)
$$

where $\epsilon_{S U M}$ is normalized by $S U M\left(H_{0}\right)$.

Keeping in mind that $k=\epsilon_{M} \cdot N$, we obtain the relationship between the error objective of H-GAP and the error objective of the monitoring function $S U M$ :

$$
\epsilon_{M}=\frac{S U M\left(H_{0}\right)}{N \cdot \Delta w} \cdot \epsilon_{S U M}
$$

Observe that our approach leads to a linear relationship between the two error objectives, which will be validated through simulation in Section IX. We call $\lambda=\frac{S U M\left(H_{0}\right)}{N \cdot \Delta w}$ the proportionality factor between $\epsilon_{M}$ and $\epsilon_{S U M}$.

Note that in an implementation scenario, $N$ and $k$ are readily available at the root node through $H_{0}$, while $\Delta w$ can be estimated in real time, during the monitoring process, or predicted from the stochastic process $\mathcal{P}$ governing the local value change [1].

\section{Simulation Results}

\section{A. Simulation Setup}

We evaluate our proposed protocol through extensive simulations using the SIMPSON simulator [29]. We use five static network topologies containing $N=82,164,328,654$ and 1308 nodes respectively, generated by GoCast [30] with a target connectivity of 5 . The aggregating trees built on top of these topologies have a maximum out-degree of 6 . The local management variable in the simulation experiments represents the number of HTTP flows traversing a given node. The monitored aggregate is the histogram of the variable in the network. The local variables are updated asynchronously based on packet traces captured on two 1Gbit/s links that connect the University of Twente to a research network [31]. We use these traces in order to create events that are processed by the simulator. Each event changes the local variable of a node at a given time. All simulations start with a protocol initialization period during which our protocol constructs the aggregation tree, Algorithm 1 initializes, and each parent node computes an initial estimate $\hat{R_{n}^{i n}}$. Specific simulation parameters are presented in Table II.

\section{B. Scalability, Controllability and Accuracy}

First, we evaluate the scalability of H-GAP by computing the maximum link utilization in all network scenarios, for various error objectives. Link utilization is computed as the 


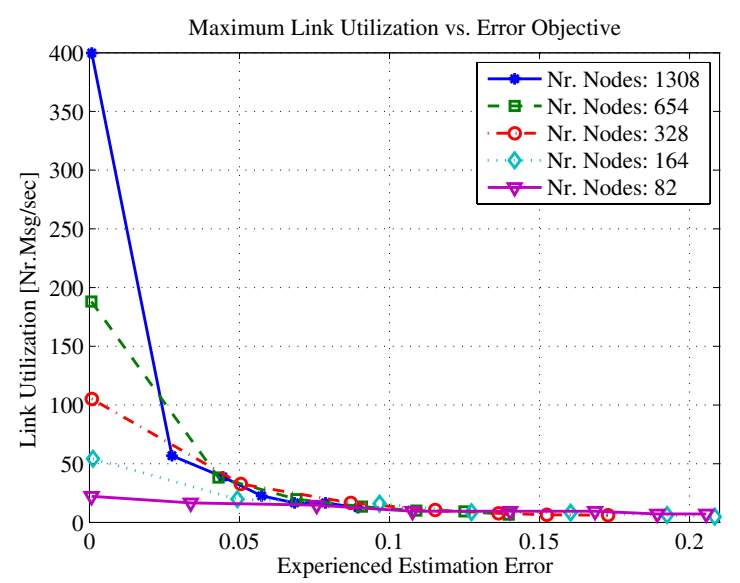

Fig. 2. Maximum link utilization vs. experienced error $\bar{\epsilon}$.

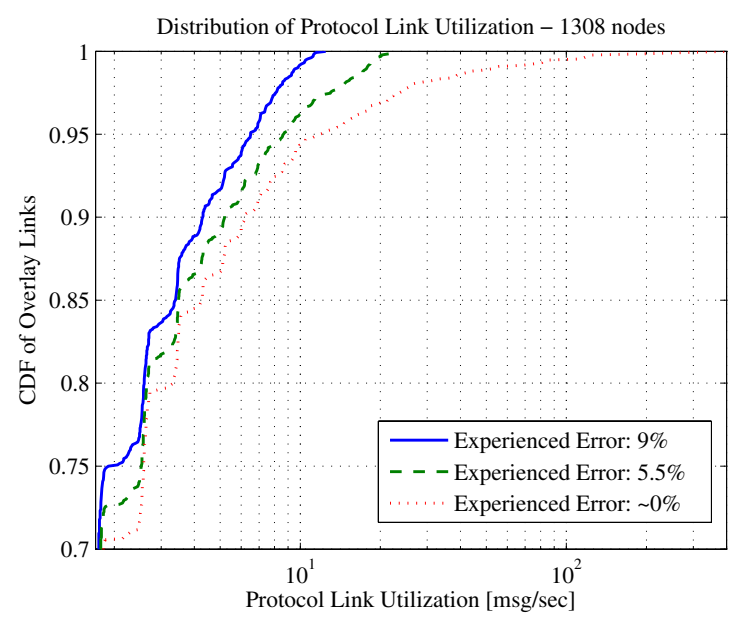

Fig. 3. Distribution of management protocol overhead on overlay links 1308 nodes.

number of messages per second exchanged by our protocol on a given link ${ }^{2}$, averaged over the monitoring time (Figure 2). We observe that even for large networks, a minimum increase in the error objective leads to a dramatic reduction in link utilization. For error objectives above $5 \%$ the maximum link utilization is approximately the same regardless of the network size. Such result has been expected as the protocol distributes the largest error-shares to nodes closer to the root where the link utilization is higher. At the same time, H-GAP always allocates an available error-share to the child that produces the maximum protocol overhead (hence the largest link utilization), trying to decrease it. The cumulative distribution function of the link utilization for the entire management overlay for two network scenarios is presented in Figure 3 and Figure 4. The results confirm the drastic reduction in variability in terms of link utilization for the management aggregation process. These results prove that our protocol scales well with increased network size which, together with its low computational complexity, makes it a suitable candidate for the monitoring of large-scale networks.

Next, we are interested in the controllability of our protocol,

\footnotetext{
${ }^{2}$ As the message size is considered as constant in our work, the mapping to a link utilization metric based on bytes per second is straightforward.
}

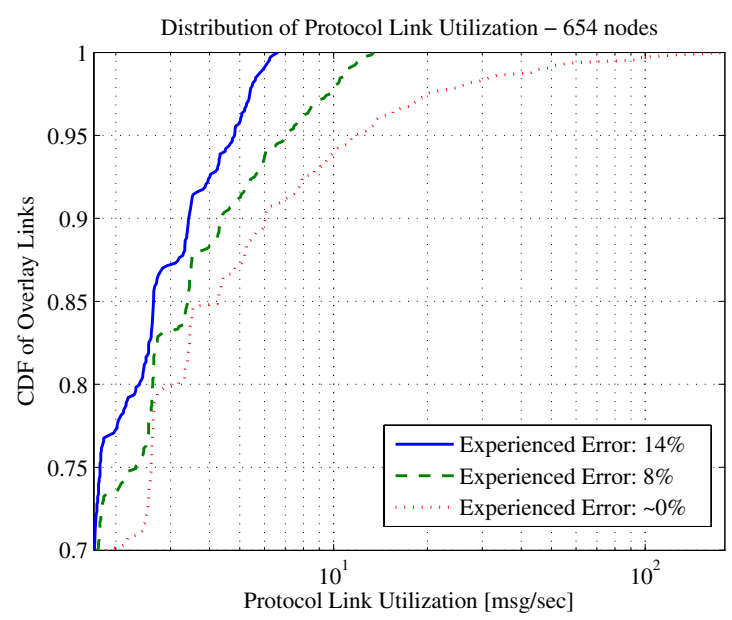

Fig. 4. Distribution of management protocol overhead on overlay links 654 nodes.

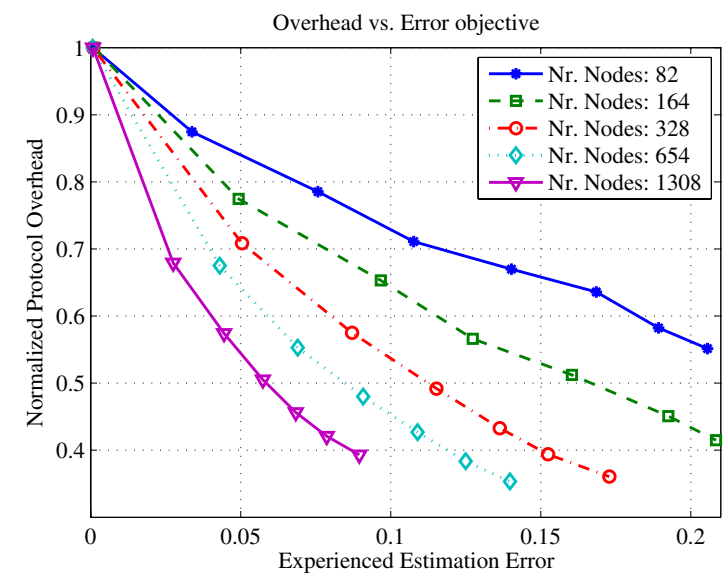

Fig. 5. Normalized protocol overhead vs. experienced error $\bar{\epsilon}$.

and we test the reduction in protocol overhead when we increase the error objective for all simulation scenarios. We measure the total number of forwarded messages in the aggregation tree during the monitoring time. We do not count the leaf updates to their parents as they are not affected by the error objective (Section VI). Figure 5 presents the obtained results normalized with the total number of messages exchanged for an error objective $\epsilon_{M}=0$. We observe a significant drop in total protocol overhead even for small error objectives. It is also noticeable that the performance of our protocol increases for larger networks, as these topologies offer more flexibility in error-share allocation.

Finally we are interested in comparing the accuracy of the protocol's error objective compared to the real error in the

obtained results. With this respect, we test our protocol for various pre-set error objectives and we compute the actual aggregation error at the root node. The aggregation error is computed periodically as the distance between the current aggregated histogram available at the root node, and the real histogram of the local variables computed from the available traces. Figure 6 and Figure 7 present the results for two network scenarios, while observed results for all other scenarios are similar. We observe a small under-valuation of 


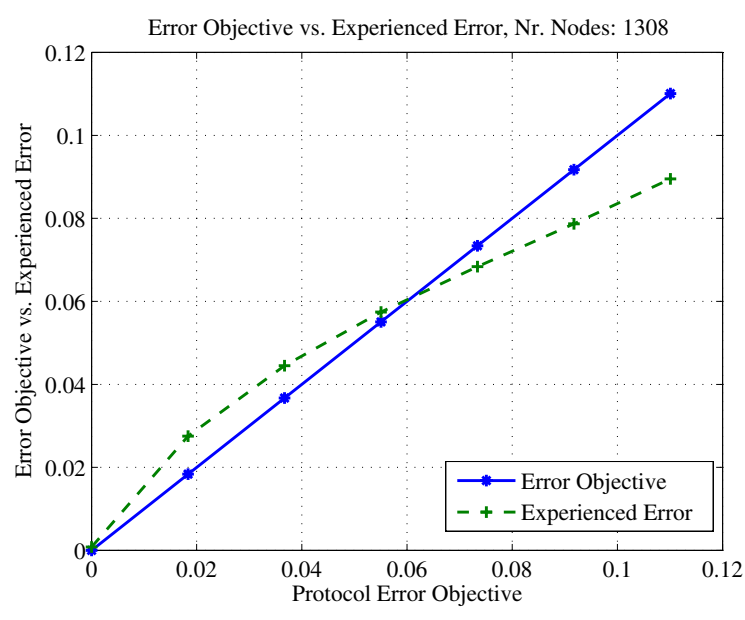

Fig. 6. Error objective $\overline{\epsilon_{M}}$ vs. experienced error $\bar{\epsilon}-1308$ nodes.

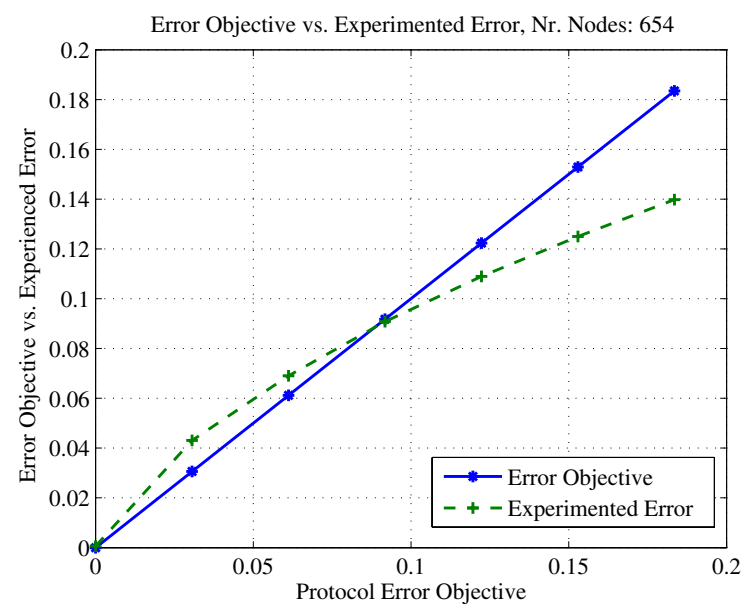

Fig. 7. Error objective $\overline{\epsilon_{M}}$ vs. experienced error $\bar{\epsilon}-654$ nodes.

the total error for extremely low error objectives, while the real error tends to be smaller than the pre-set error objectives for higher values. This is explained by the fact that for higher error-objectives we observe the canceling effect of histogram aggregation at intermediate nodes. Parents wait for more child updates before forwarding the aggregate up the tree, hence increasing the chance of observing updates with changes that cancel each other out. This effect cannot be observed in asynchronous systems at low error objectives, as intermediate nodes must forward almost all incoming updates without waiting for more updates that might cancel some of the changes.

\section{Error Objective Mapping for SUM and Comparison to A-GAP}

The final results presented in this paper reflect the accuracy of the error mapping for the management function $S U M$, as presented in Section VIII. We use a modified version of our original trace of local weights change, in order to introduce more variability in the global aggregate time-evolution [32]. To the original trace at process $i$, we add a sinusoidal bias:

$$
w_{i}^{\prime}(t)=\operatorname{int}\left(w_{i}(t)+23 \cdot\left[1+\sin \left(\frac{2 \pi t}{30}-\frac{\pi}{2}\right)\right]\right),
$$

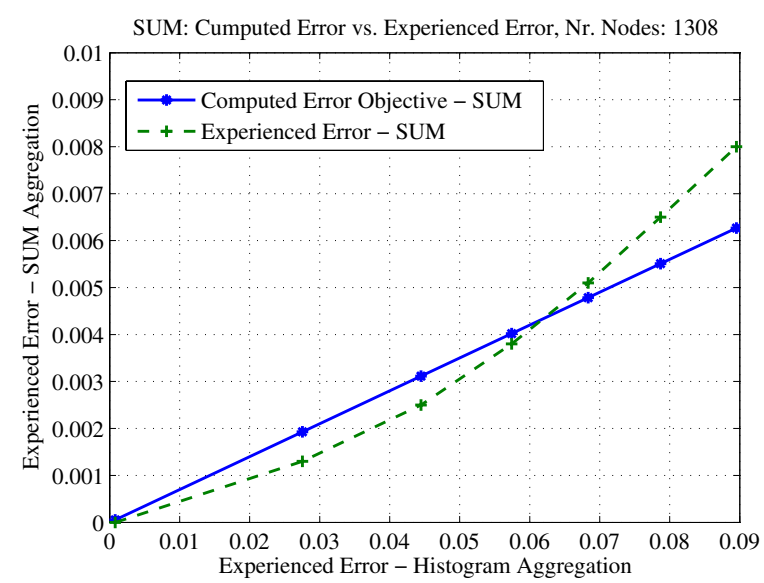

Fig. 8. Computed vs. experienced error for management function $S U M$ 1308 nodes.

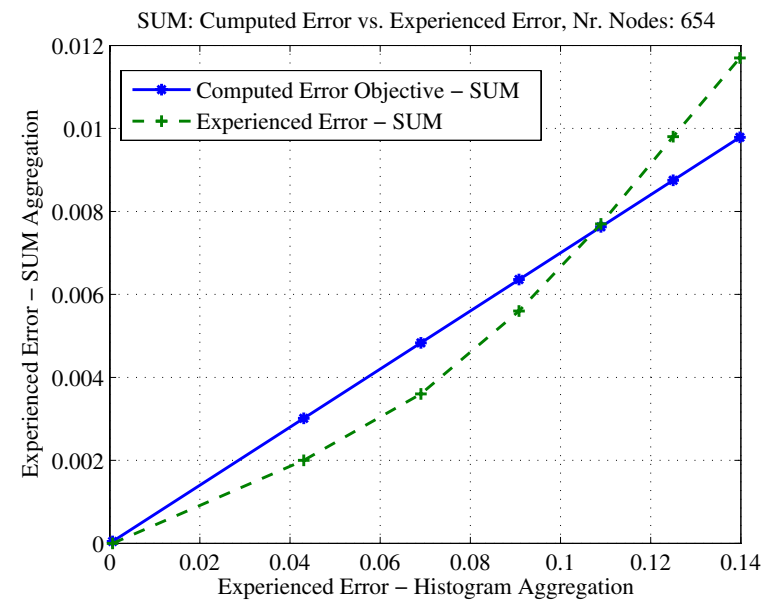

Fig. 9. Computed vs. experienced error for management function $S U M$ 654 nodes.

where $\operatorname{int}()$ returns the integer component of its argument.

The average computed proportionality factor for this trace is $\lambda=14.28$. Comparison results between the computed error mapping from $\epsilon_{M}$ to $\epsilon_{S U M}$ for two network scenarios are presented in Figure 8 and Figure 9.

We observe that the experienced error while computing $S U M$ on the aggregated histogram follows the general behavior as described by our proposed mapping. However, since our mapping is only based on the monitoring of variable change, and does not use any estimation/prediction tools, it tends to be rather inexact. We conclude that while our histogram aggregation protocol brings gains in the flexibility of applying any function on the data set at the root node, it suffers in terms of prediction accuracy for the final experienced error, for a specific management function result.

Finally, we compare our proposed mechanisms to A-GAP, a specialized protocol for aggregating $S U M$ [1]. We test $\mathrm{H}$ GAP in the same simulation conditions as presented in [1], and evaluate the performance of our protocol in terms of the trade-off: maximum processing node-load versus average error in terms of number of HTTP flows estimated at the root node. We use the same computation procedure as in the case of the A-GAP protocol. A zoomed-in snapshot of the results is 


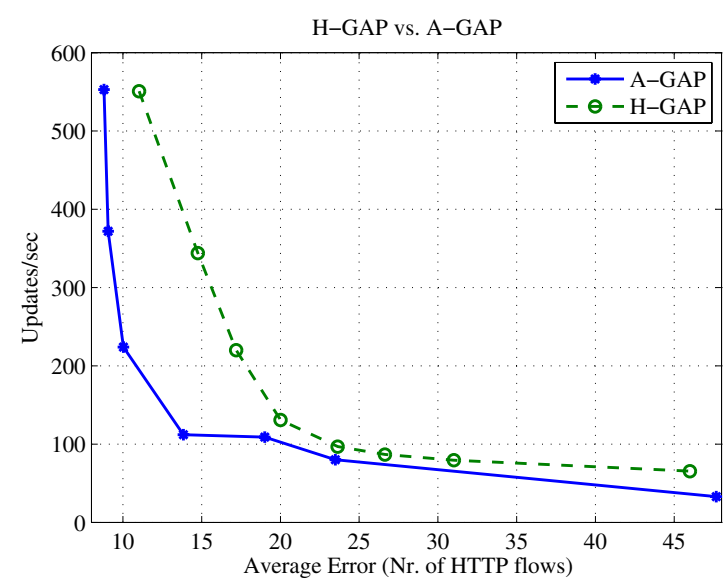

Fig. 10. Performance comparison: H-GAP vs. A-GAP.

presented in Figure 10, and shows a similar behavior for both protocols. A-GAP is more efficient especially for very low error objectives (i.e., 10-20 HTTP flows out of an average of 20.000 HTTP flows in total, hence an error objective of below $0.1 \%$ ), as it is specialized for the given management function. However, H-GAP offers the advantage of flexibility at the root node in switching the management function, and is much faster, as it is based on linear-time error-share allocation algorithms.

\section{DISCUSSION}

\section{A. The efficiency of estimating SUM using $H$-GAP}

Our simulation results show that for a given error objective $\epsilon_{S U M}$, the corresponding error objective $\epsilon_{M}$ for H-GAP is more than one order of magnitude larger, which translates into a low protocol overhead for both considered metrics. This result is of course dependent on the particular trace used for the evolution of local variables.

Further insight beyond this particular trace is provided by the model introduced in Section VIII for the mapping of $\epsilon_{S U M}$ to $\epsilon_{M}$. This mapping is expressed through the linear relationship $\epsilon_{M}=\lambda \cdot \epsilon_{S U M}$, with the proportionality factor $\lambda=\frac{S U M\left(H_{0}\right)}{N \cdot \Delta w} \cdot \lambda$ can be expressed as $\frac{\bar{w}}{\Delta w}$, where $\bar{w}$ stands for the average value of all local weights. The proportionality factor is a measure inversely proportional to the relative variability of the trace. The above mapping function suggests that the smaller the relative variability of the trace, the larger the value of $\epsilon_{M}$ given an $\epsilon_{S U M}$, and therefore the lower the protocol overhead in estimating $S U M$ for a given accuracy objective. While this mapping is instrumental in predicting the performance of our protocol for other traces, it should be further validated with additional experiments.

\section{B. Optimization criterion}

H-GAP represents our distributed solution for histogram aggregation over aggregation trees with an accuracy objective. We define the objective as the maximum tolerated distance between the protocol-generated histogram and the actual value distribution in the system. Given this error objective, we formulate the optimization problem as to minimize the maximum link load imposed by the protocol on the network graph. Such an optimization function is appropriate for instance for a sensor network scenario, where the total lifetime of the system often depends on the node with the shortest lifetime based on its local energy resources.

Alternatively, we can define the optimization problem as to minimize the total number of required protocol messages on the network graph. Such a choice can be appropriate for traditional wireline networks, where the resources of the management plane are minimized for a given operation. In this case, the global optimization problem is modified in order to reflect the change in the optimization criterion. If $\Omega_{n}(t)$ is the total overhead observed at node $n$, then the global optimization problem in Eq. (3) is reformulated as:

$$
\left.\epsilon(\vec{t})^{*}=\underset{\forall \overrightarrow{(\epsilon(t))}}{\arg \min } \sum_{n=0}^{N} \Omega_{n}\left(t, \mathcal{P}, \epsilon_{j}(t)\right)\right),
$$

while keeping the same constraint in place.

It turns out that we can use the same distributed heuristic to produce good solutions for both instances of the global optimization problem. In Section IX we evaluate H-GAP with respect to both optimization metrics, i.e., maximum link utilization and total number of messages in the system. We observe that H-GAP is efficient with respect to both objectives.

\section{Node computational cost vs traffic overhead}

In the presented optimization problems we are expressing the protocol overhead in terms of messages sent and received in order to aggregate the histogram of local values.

As an alternative, one can consider protocol overhead in terms of computational resources required in the management processes for aggregation. Applying a simple mapping between the resources needed to process a message and to send/receive a message, the above optimization problems can be interpreted both in terms of minimizing message overhead, as well as minimizing computational overhead.

\section{Controlling the trade-off between message size and number of updates through quantization}

Notice that our analysis and solution take into account only real-valued local variables with a finite value space $S$. Our protocol can be extended for the monitoring of variables over infinite sets $S$ by incorporating an initial quantization step. Local values can be quantized with a quantization step $Q$. The size of $Q$ determines the size of the aggregation vector $H$ and hence the size of the exchanged messages in the system.

In this case, the global optimization problem can be formulated in terms of the total amount of exchanged data/bytes instead of the number of exchanged messages. Furthermore we consider a joint error objective $\epsilon_{M}$ which consists of the quantization error $\epsilon_{Q}$ (which influences the quantization step $Q$ and the size of messages) and the aggregation error $\epsilon_{\tau}$ (which influences the rate of updates propagated up the tree).

The combined optimization of both aspects of the monitoring process, namely quantization and aggregation, can be modeled as a joint source-channel coding problem. An advantage of such a perspective would be that the trade-off 
between message size and the number of messages becomes controllable in the aggregation protocol. The investigation of this problem could bring meaningful interdisciplinary parallels and is left for future work.

\section{CONCLUSIONS}

In this paper we present H-GAP, a distributed protocol for the aggregation of the histogram of a monitored local variable in large-scale systems. Our protocol provides the management station with timely information about the distribution of the values of the monitored variable with minimal protocol overhead, while satisfying a global accuracy objective. HGAP is tunable, in the sense that the operating point on the trade-off curve (between protocol overhead and accuracy objective) can be controlled at runtime. Based on the aggregated histogram, the management station can compute various aggregation functions, either sequentially or in parallel. This is not possible with traditional aggregation protocols, which integrate a specific aggregation function with the monitoring process.

Our contribution includes a detailed analysis of the problem of histogram aggregation over aggregation trees, a formulation of the global optimization problem, and a complete distributed solution containing heuristic, tree-based algorithms suitable for real system implementation. Furthermore, we provide an efficient solution for the mapping of the error objective of a general aggregation function to the error objective of H-GAP, using $S U M$ as an example. To the best of our knowledge, we are the first to present a complete protocol for efficiently aggregating histograms under error objectives, and to show how such a protocol can be effectively used to compute general aggregation functions with specific error objectives. Lastly, we validate the protocol design objectives through simulations using real traces.

From the obtained results we draw two main conclusions. First, the effectiveness and efficiency of H-GAP increase with the system size, in the sense that, in larger settings, a given increase in the error objective leads to an increasing reduction in the protocol overhead. Second, the trade-off curve between accuracy objective and protocol overhead suggests the existence of a favorable operating point, for which $\mathrm{H}$ GAP provides high accuracy for a comparably low overhead. As part of future work, we plan to extend the protocol in such a way that it auto-configures to reach and maintain this particular operating point.

\section{REFERENCES}

[1] A. Gonzalez and R. Stadler, "A-GAP: An adaptive protocol for continuous network monitoring with accuracy objectives," IEEE Trans. Netw. Service Management, vol. 4, no. 1, pp. 2-12, June 2007.

[2] G. Cormode, M. Garofalakis, S. Muthukrishnan, and R. Rastogi, "Holistic Aggregates in a networked world: Distributed tracking of approximate quantiles," in Proc. ACM SIGMOD, June 2005.

[3] A. Deligiannakis, Y. Kotidis, and N. RoussoPoulos, "Hierarchical innetwork data aggregation with quality guarantees," in Proc. EDBT Crete, Greece, Mar. 2004.

[4] R. van Renesse, K. Birman, and W. Vogels, "Asltrolabe: A robust and scalable technology for distributed system monitoring," ACM Trans. Comput. Syst., vol. 21, no. 2, pp. 164-206, May 2003.

[5] M. A. Sharaf, J. Beaver, A. Labrinidis, and P. K. Chrysantis, "Balancing energy efficiency and quality of aggregate data in sensor networks," ACM International J. Very Large Data Bases, vol. 13, no. 4, pp. 384403, Dec. 2004
[6] C. Intanagonwiwat, R. Govindan, and D. Estrin, "Directed diffusion: A scalable and robust communication paradigm for sensor networks," in Proc. ACM MOBICOM, Boston, USA, Aug. 2000.

[7] B. Krishnamaachari, D. Estrin, and S. Wicker, "The impact of data aggregation in wireless sensor networks," in Proc. International Workshop Distributed Event-Based Systems, Vienna, Austria, July 2002.

[8] S. R. Madden, M. J. Franklin, J. M. Hellerstein, and W. Hong, "TAG: A tiny aggregation service for ad-hoc sensor networks," in Proc. Fifth Symp. Operating Syst. Design Implementation, Boston, USA, Dec. 2002.

[9] I. Solis and K. Obraczka, "The impact of timing in data aggregation for sensor networks," in Proc. International Conf. Commun. ICC, Paris, France, June 2004.

[10] H. Singhal and G. Michailidis, "Optimal sampling in state space models with applications to network monitoring," in Proc. 2008 ACM SIGMETRICS, NY, USA, June 2008.

[11] C. Intanagonwiwat, R. Govindan, and D. Estrin, "Directed diffusion: A scalable and robust communication paradigm for sensor networks," in Proc. Sixth Annual International Conf. Mobile Comput. Netw. (MOBICOM), Boston, USA, Aug. 2000.

[12] B. Krishnamachari, D. Estrin, and S. Wicker, "Modelling data-centric routing in wireless sensor networks," UCS - Computer Engineering, TR - CENG 02-14, 2002.

[13] _ - "The impact of data aggregation in wireless sensor networks," in Proc. International Workshop Distributed Event-Based Syst., Vienna, Austria, July 2002.

[14] S. R. Madden, M. J. Franklin, J. M. Hellerstein, and W. Hong, "TAG: A tiny aggregation service for ad-hoc sensor networks," in Proc. Fifth Symp. Operating Syst. Design Implementation, Boston, USA, December 2002.

[15] C. Intanagonwiwat, D. Estrin, R. Govindan, and J. Heidemann, "Impact of network density on data aggregation in wireless sensor networks," in Proc. 22nd International Conf. Distributed Comput. Syst., Vienna, Austria, July 2002.

[16] M. A. Sharaf, J. Beaver, A. Labrinidis, and P. K. Chrysanthis, "Balancing energy efficiency and quality of aggregate data in sensor networks," ACM International J. Very Large Data Bases, vol. 13, no. 4, pp. 384403, Dec. 2004

[17] A. Boulis, S. Ganeriwal, and M. B. Srivastava, "Aggregation in sensor networks: An energy - accuracy trade-off," Elsevier Ad-Hoc Networks J. (special issue sensor netw. protocols applications), pp. 317-331, 2003.

[18] C. Olston, B. T. Loo, and J. Widom, "Adaptive precision setting for cached approximate values," in Proc. ACM SIGMOD, Santa Barbara, USA, May 2001.

[19] C. Olston and J. Widom, "Efficient monitoring and querying of distributed, dynamic data via approximate replication," in Proc. IEEE Data Enigneering Bulletin, Mar. 2005.

[20] C. Olston, J. Jiang, and J. Widom, "Adaptive filters for continuous queries over distributed data streams," in Proc. ACM SIGMOD, San Diego, USA, June 2003.

[21] A. Gonzalez, "Adaptive management for networked systems," Licensiate Thesis, School of Electrical Engineering, KTH Royal Institute of Technology, Sweden, Tech. Rep., June 2006.

[22] Y. Tang, S. Chen, and Y. Ling, "State aggregation of large network domains," Comput. Commun., vol. 30, no. 4, pp. 837-885, 2007.

[23] D. Kempe, A. Dobra, and J. Gehrke, "Gossip-based computation of aggregate information," in Proc. 44th Annual IEEE International Symp. Foundations Comput. Science, Boston, USA, Oct. 2003.

[24] M. Haridasan and R. van Renesse, "Gossip-based distribution estimation in peer-to-peer networks," in Proc. Seventh International Workshop Peer-to-Peer Syst., FL, USA, Feb. 2008.

[25] F. Wuhib, M. Dam, R. Stadler, and A. Clemm, "Robust monitoring of network-wide aggregates through gossiping," Trans. Netw. Service Management (TNSM), vol. 6, no. 2, June 2009.

[26] W. Rudin, Real and Complex Analysis 3rd ed., WBC/McGraw-Hill, Ed. The McGraw-Hill Series in Higher Mathematics, 1987.

[27] M. Dam and R. Stadler, "A Generic protocol for network state aggregation," in Proc. Radiovetenskap och Kommun. (RVK), Linkoping, Sweden, June 2005.

[28] IEEE, "ANSI/IEEE Std 802.1D," IEEE, 1998 Edition.

[29] K. Lim and R.Stadler, "SIMPSON - a SIMple pattern simulator for networks," [Online]. Available: http://www.s3.kth.se/lcn/software/simpson.shtml, July 2006.

[30] C. Tang and C. Ward, "GoCast: Gossip-enhanced overlay multicast for fast and dependable group communication," in Proc. International Conf. Dependable Syst. Netw. (DSN'05), Yokohama, Japan, June 2005.

[31] R. van der Meent and A. Pras, "Traffic measurement data repository, University of Twente," [Online]. Available: http://trafficrepository.ewi.utwente.nl, May 2006. 
[32] F. Wuhib, M. Dam, and R. Stadler, "Decentralized detection of global threshold crossings using aggregation trees," Comput. Netw., vol. 52, no. 9 , pp. 1745-1761, 2008.

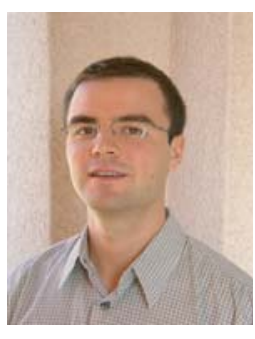

Dan Jurca received his M.S. degree from the "Politehnica" University of Timsoara, Romania in 2002. In 2007 he received his Ph.D. degree in electrical engineering from the Swiss Federal Insitute of Technology (EPFL), Lausanne, Switzerland, with his main topic of research focused on real-time adaptive multimedia streaming. Between 2007 and 2008 he worked as a post doctoral researcher in the Royal Institute of Technology (KTH), Stockholm, Sweden, on distributed network management aspects. He is currently working as a researcher for DOCOMO Communications Laboratories Europe $\mathrm{GmbH}$, Munich, Germany, inside the Ubiquitos Networking Research Group. His research interests focus on reconfigurable mobile networks, multimedia streaming and QoE, and application and user mobility.

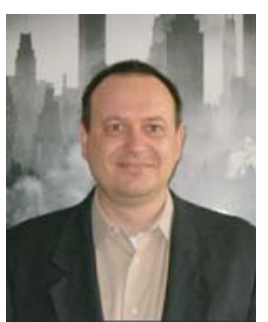

Rolf Stadler (www.ee.kth.se/ stadler) is a professor at the Royal Institute of Technology (KTH), Stockholm, Sweden. He holds a M.Sc. degree in mathematics and a Ph.D. in computer science, both from the University of Zurich. In 1991 he was a researcher at the IBM Zurich Research Laboratory. From 1992 to 1994 he was a visiting scholar at Columbia University in New York, which he joined in 1994 as a research scientist. From 1998 to 1999 he was a visiting professor at ETH Zurich. He joined the faculty of KTH in 2001 and is now at the School of Electrical Engineering, where he leads research into the management of networked systems. 